\title{
Gfi1.1 regulates hematopoietic lineage differentiation during zebrafish embryogenesis
}

Wei Wei ${ }^{1}$, Lu Wen ${ }^{1}$, Peng Huang ${ }^{1}$, Zheng Zhang ${ }^{1}$, Yuanyuan Chen ${ }^{1}$, An Xiao ${ }^{1}$, Haigen Huang ${ }^{2}$, Zuoyan Zhu ${ }^{1}$, Bo Zhang $^{1}$, Shuo Lin ${ }^{1,2}$

${ }^{1}$ Key Laboratory of Cell Proliferation and Differentiation, Center of Developmental Biology and Genetics, College of Life Sciences, Peking University, Ministry of Education, Beijing 100871, China; ${ }^{2}$ Department of Molecular, Cell \& Developmental Biology, University of California, Los Angeles, CA 90095-1606, USA

Growth factor independence 1 (GFI1) is important for maturation of mammalian lymphocytes and neutrophils and maintenance of adult hematopoietic stem cells (HSCs). The role of GFI1 in embryonic hematopoiesis is less well characterized. Through an enhancer trap screen and bioinformatics analysis, we identified a zebrafish homolog of Gfil (named $g$ fil.1) and analyzed its function during embryonic development. Expression of both an endogenous gfil.1 gene and a $G F P$ reporter gene inserted near its genomic locus was detected in hematopoietic cells of zebrafish embryos. Morpholino (MO) knockdown of gfil.1 reduced expression of scl, Imo2, c-myb, mpo, rag1, gata1 and hemoglobin alpha embryonic-1 (hbae1), as well as the total amount of embryonic hemoglobin, but increased expression of pu.1 and l-plastin. Under the same conditions, MO injection did not affect the markers involved in vascular and pronephric development. Conversely, overexpression of gfil.1 via mRNA injection enhanced expression of gatal but inhibited expression of pu.1. These findings suggest that Gfi1.1 plays a critical role in regulating the balance of embryonic erythroid and myeloid lineage determination, and is also required for the differentiation of lymphocytes and granulocytes during zebrafish embryogenesis.

Keywords: gfil.1, hematopoiesis, zebrafish

Cell Research (2008) 18:677-685. doi: 10.1038/cr.2008.60; published online May 272008

\section{Introduction}

Growth factor independence 1 (Gfil) was initially identified in a retroviral insertional mutagenesis screen for tumor cell proliferation that was independent of the interleukin-2 growth factor [1]. GFI1 is widely conserved as its related proteins have been identified from many species including Drosophila, Caenorhabditis elegans, zebrafish (Danio rerio), mice and humans. The human GFI1 protein contains a domain consisting of six $\mathrm{C}$-terminal $\mathrm{C} 2 \mathrm{H} 2$ zinc fingers for DNA binding, a 20-amino-acid N-terminal SNAG domain for mediating transcriptional repressor activity and a conserved nuclear localization signal (NLS). These domains are highly conserved, although the middle region

Correspondence: Bo Zhang ${ }^{\mathrm{a}}$, Shuo Lin ${ }^{\mathrm{b}}$

${ }^{a}$ Tel/Fax: +86-10-6275-9072; E-mail: bzhang@pku.edu.cn

${ }^{b}$ Tel: +01-310-267-4970; Fax: +01-310-267-4971; E-mail: shuolin@ucla. edu

Received 13 September 2007; revised 11 November 2007; accepted 7 December 2007; published online May 272008 of GFI1 protein is more variable among different species [1-3]. Mouse Gfil and human GFI1 are expressed exclusively in hematopoietic stem cells (HSCs) and the immune system, and mouse GFI1 functions as a transcriptional regulator in lymphoid cells $[1,4]$. Previous studies have shown that mouse Gfil can act as an oncogene when overexpressed and can cooperate with other oncoproteins such as PIM-1 and MYC in promoting T-cell lymphomagenesis [5-8]. Mouse GFI1 also regulates IL-4/STAT6-dependent Th2 cell proliferation [9] and IL-6/STAT3-mediated cell proliferation in response to antigenic stimulation $[9,10]$. Genetic studies have shown that mouse Gfil is important for neutrophil maturation [11] and that mice lacking Gfil are neutropenic owing to their failure to repress Ela2 [12, 13]. In these mice, the differentiation of both neutrophils and $\mathrm{T}$ lymphocytes is blocked [14]. Recent analyses have established that GFI1 plays critical roles in regulating self-renewal and maintaining adult HSCs [15-17]. Mice deficient in Gfil exhibit altered proliferation of HSCs in their bone marrow, which is probably due to abnormal cell cycle through deregulation of the interaction between 
GFI1B, a protein closely related to GFI1, and $\mathrm{p} 21^{\text {Cipl/wafl }}$ $[15,16]$. These studies indicate that GFI1 plays multiple roles during hematopoiesis, including self-renewal, multilineage differentiation, and lineage commitment and terminal differentiation. Despite the progress that has been made by these studies, the role of GFI1 during embryonic hematopoiesis has not been well studied.

We seek to study Gfil function in embryogenesis using zebrafish as a model. Zebrafish is ideal for analyzing hematopoiesis owing to its rapid external embryonic development and transparency. Zebrafish hematopoiesis can be visualized in live embryos using transgenic fluorescent protein reporters and embryos can survive for several days without circulating blood cells [18]. Studies in zebrafish have established that many genes critical for mammalian hematopoiesis are conserved, and their tightly regulated spatial-temporal expression leads to the balanced differentiation of different blood cells during zebrafish development $[19,20]$. However, it seems that Gfil is not included as one of these genes even though a homolog of Gfil has been identified in zebrafish [21]. The likely reason is that this gene is not the true zebrafish ortholog of mammalian Gfil since its expression is not in hematopoietic cells but appears prominently in neural tissues. Recently we performed an independent large-scale enhancer trap screen based on the Tol2 transposon in zebrafish with the aim of identifying tissue-specific genes and generating their transgenic fluorescent protein reporter lines. Through this screen we identified more than 30 lines of transgenic zebrafish that exhibit hematopoietic expression of a green or red fluorescent protein reporter gene (our unpublished data). Molecular analyses of these fish revealed that one of the enhancer trap lines carries an insertion near a new Gfil homologous gene.

To avoid conflict in the nomenclature with the previously reported zebrafish gfil gene, we designated this new Gfil homolog as gfil.1. In this study, we showed that zebrafish gfil.1 shares high sequence homology and a good genomic synteny with mouse Gfil and human GFI1. The embryonic expression of $g$ fil.1 is restricted to the hematopoietic tissues in zebrafish. More importantly, gfil.1 functions in promoting differentiation of the erythroid while repressing myeloid lineage determination; it is also required for the differentiation of lymphocytes and granulocytes during zebrafish embryogenesis. Our studies therefore established a critical developmental role for $g$ fil. 1 during hematopoiesis in a non-mammalian vertebrate species.

\section{Results}

\section{Identification of zebrafish gfil.1}

Our enhancer trap screen generated approximately 1700 transgenic zebrafish lines with GFP expressed in various organs, including more than 30 lines expressing GFP specifically in hematopoietic tissues (our unpublished data). Sequence analyses of insertion sites by linker-mediated PCR revealed that one of these lines trapped a gene designated as NM_001020776.1 in GenBank or zgc:113277 in Ensembl at approximately $20 \mathrm{~kb}$ upstream of its putative transcription start site. This gene encodes a protein of 385 amino-acid residues $(\sim 47 \mathrm{kDa})$ which shares significant homology with mouse Gfi1 and human GFI1. Since Dufourcq et al. previously already reported a different zebrafish gene as $g f i 1$ as being expressed in the ganglion cells of the neural retina and in developing hair cells of the ear [21], we named the gene revealed by our enhancer trap screen as gfil.1.

Mammalian GFI1 proteins have an evolutionally conserved SNAG domain at the N-terminus and a cluster of six zinc-finger motifs at the C-terminus. Zebrafish Gfil.1 protein also contains a SNAG domain with $95 \%$ similarity and a six zinc-finger domain with $98 \%$ similarity to those in humans (Figure 1A). Phylogenetic analysis using the conserved zinc-finger domain sequences also suggests that Gfil.1 has a close relationship in evolution with the mammalian GFI1 proteins (Figure 1B). Zebrafish gfil.1 is located on chromosome 2 , where its syntenic neighboring genes are also conserved in the chromosomal region around human GFII on chromosome 1 and the chromosomal region around mouse Gfil on chromosome 5 (Figure 1C). Interestingly, the previously reported zebrafish $g f i l$ gene that is on chromosome 6 also shows similar syntenic conservation to its mammalian homologs (Supplementary information, Figure S1), which suggests that there is a duplication of this chromosome region in the zebrafish genome.

\section{Expression of gfil.1 and GFP in gfil.1:GFP transgenic embryos}

To detect the transcriptional activities of zebrafish $g$ fil.1 during early embryonic development, we first analyzed gfil.1 mRNA levels in 0 to 5 days post fertilization (dpf) embryos using real time quantitative PCR (RT-qPCR). Initially, $g$ fil.1 showed a high level of maternal expression, which gradually decreased to approximately $10 \%$ of the maternal level at $48 \mathrm{hpf}$ (hours post fertilization) (Figure 2A). To further investigate the tissue-specific expression pattern of $g$ fil.1, RNA whole-mount in situ hybridization was performed. Consistent with the RT-PCR result, $g$ fil.1 mRNA could be detected in the one-cell stage embryo and distributed evenly in the zygote until the late gastrula stage (Figure 2B). At approximately $10 \mathrm{hpf}, g f i 1.1$ expression was mainly restricted to two bilateral stripes flanking the paraxial mesoderm. Weak expression was also detected in the anterior lateral mesoderm (ALM) (Figure 2B). At $24 \mathrm{hpf}$, gfil.1 expression domains converged into the intermediate cell mass (ICM), the single midline hematopoietic region, 
A
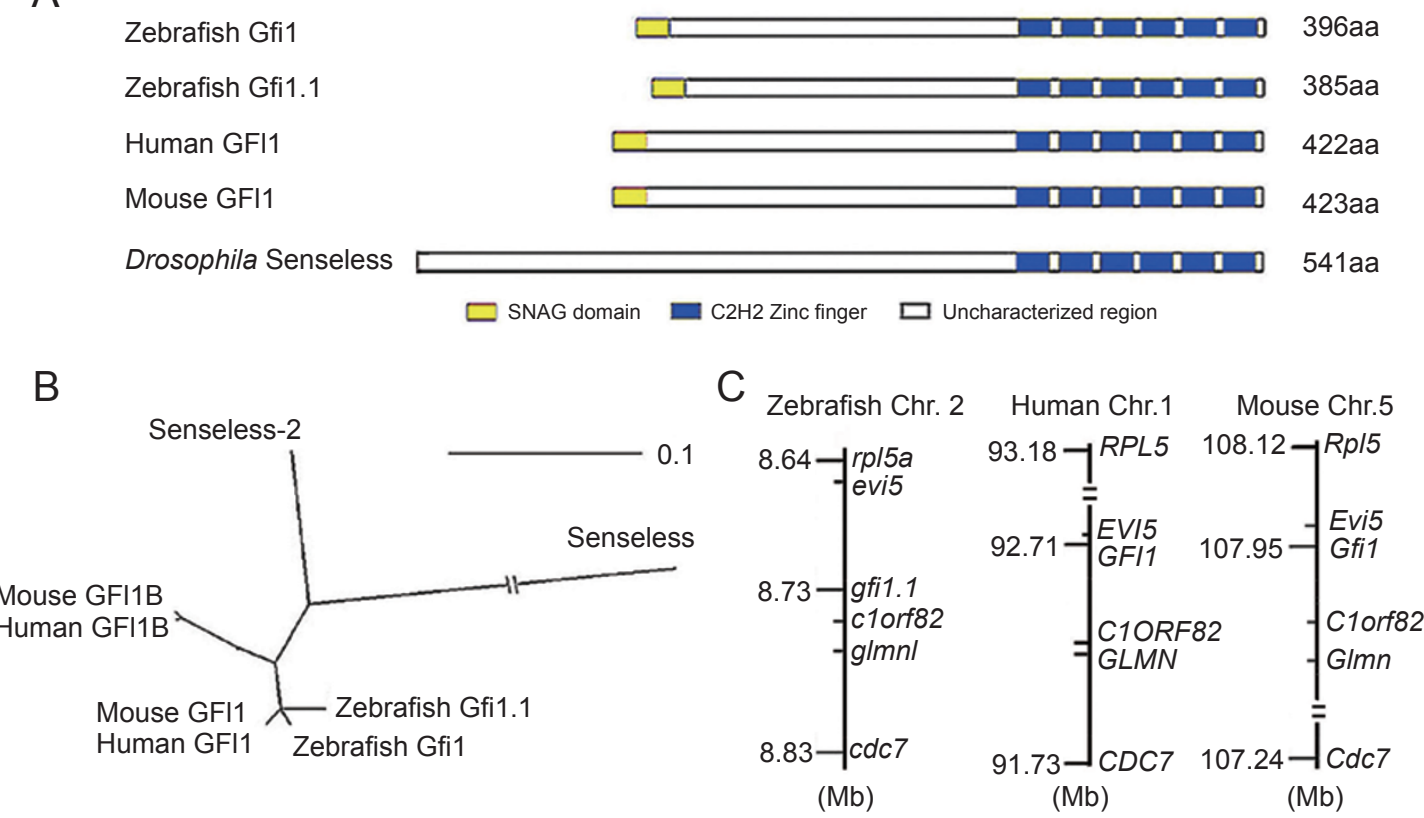

Figure 1 Identification of gfi1.1 in zebrafish. (A) Protein structure comparison of Gfi1 homologs from zebrafish, human, mouse and Drosophila (Senseless). (B) Phylogenetic tree showing evolutionary correlations among some Gfi1 family members from zebrafish, human, mouse and Drosophila (Senseless and Senseless-2). (C) Syntenic arrangement around the gfi1.1 locus on zebrafish chromosome 2 shows remarkable similarity to the locus of its human ortholog on chromosome 1 and its mouse ortholog on chromosome 5. The relative distance between genes (in Mb) is shown to scale. Mb, megabase.

A

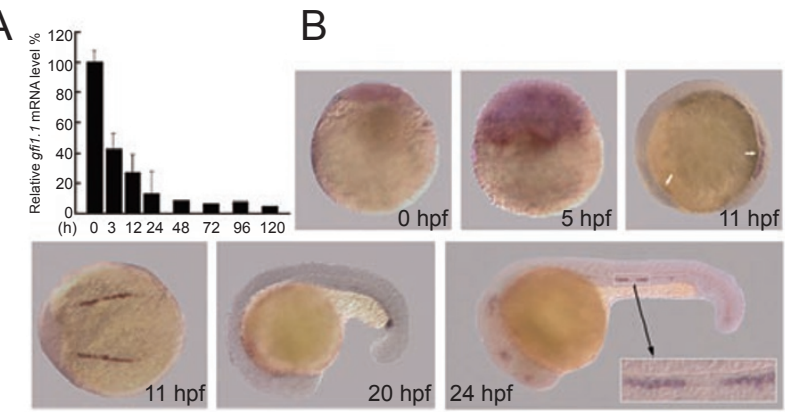

C
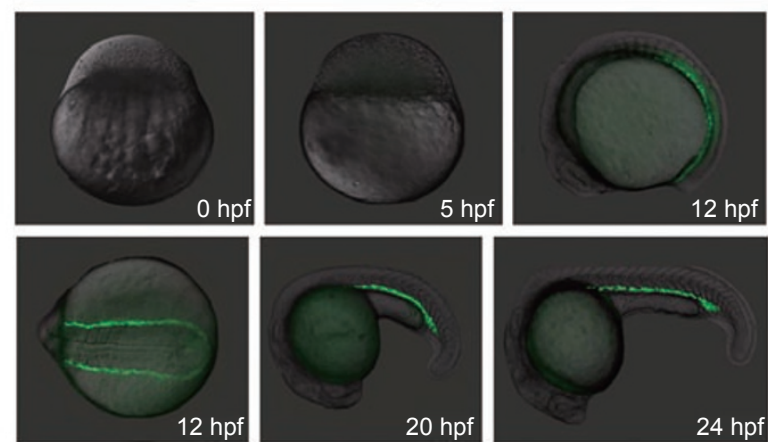

Figure 2 Expression of gfi1.1 during zebrafish embryo development. (A) Quantitative real-time PCR analysis of gfi1.1 mRNA at different stages of zebrafish embryo development. (B) Wholemount in situ hybridization of the gfi1.1 gene at the first $24 \mathrm{~h}$ of development. (C) Green fluorescent signal showing the GFP expression pattern in gfi1.1:GFP transgenic embryos. and the ears (Figure 2B). Bilateral and ICM expression is characteristic of hematopoietic marker genes such as gatal and lmo2.

Compared with the endogenous expression of $g$ fil.1, the transgenic line gfil.1:GFP lacks maternal expression of GFP (Figure 2C). At $12 \mathrm{hpf}$, GFP fluorescence was visible as two stripes that correspond to the regions of gfil.1 expression detected using RNA in situ hybridization. Later, at $24 \mathrm{hpf}$, GFP was similarly detected in the ICM and ears. The delay in appearance of the GFP fluorescence signal compared with $g$ fil.1 mRNA might reflect the time needed to accumulate enough GFP protein. Nonetheless, gfi1.1:GFP faithfully recapitulated zygotic expression of the endogenous $g$ fil.1 during embryonic development of zebrafish. In addition, the GFP pattern of gfil.1:GFP is very similar to that of gata1:GFP transgenic zebrafish, a well-characterized line that expresses GFP in early hematopoietic cells.

Functional analysis of gfil.1 in embryonic hematopoiesis

Mice deficient in Gfil are viable for 2-3 months [14-16] and it is unclear whether Gfil has any function in embryonic hematopoiesis during early development. Given that the expression of $g$ fil.1 is high in blood cells during the embryonic development of zebrafish and that Gfil has been 
found to have multiple functions in mammalian hematopoiesis, we have focused on studying the function of $g$ fil.1 in embryonic hematopoiesis using zebrafish as a model.

Antisense morpholinos (MOs) are effective in inhibiting gene function in zebrafish through blocking either protein translation or mRNA splicing [22]. In this study, two MOs to repress translation (MO1, which targets the ATG region, and $\mathrm{MO} 2$, which targets the $5^{\prime}$ UTR region) and one $\mathrm{MO}$ to block splicing (MO I1E2, which targets the junction between first intron and second exon) were used to knockdown $g$ fil.1 expression. First, several assays were carried out to validate these MOs. To test whether MO1 and MO2 could block translation of $g$ fil.1 mRNA, we performed Western blot analysis using a Gfil.1 antibody on protein extracts from embryos injected with either 4 ng MO1 or 8 ng MO2. The results showed that MO1, when injected at $4 \mathrm{ng}$ per embryo, efficiently reduced the amount of Gfil.1 protein to approximately $10 \%$ of that from uninjected controls (Supplementary information, Figure S2A, left panel), while MO2 at $8 \mathrm{ng}$ per embryo led to a $60 \%$ reduction (Supplementary information, Figure S2B). Additionally, injection of MO1 or a mixture of MO1 or MO2 with $100 \mathrm{pg}$ gfi1.1-N1 plasmid showed that translation of the gfil.1-GFP fusion gene could be effectively blocked (supplementary information, Figure S2A, right panel). Finally, the efficacy of MO I1E2 was demonstrated by a significant reduction of $g$ fil.1 mRNA in the injected embryos, as shown through RT-PCR (Supplementary information, Figure S2C).

Injection of the three MOs at a defined dose (4-8 $\mathrm{ng}$ for $\mathrm{MO} 1,8 \mathrm{ng}$ for MO2, or $10 \mathrm{ng}$ for MO I1E2) did not cause gross developmental abnormality in the embryos (embryonic survival rate: $\mathrm{MO} 1>90 \%$, MO2 $>50 \%$, MO I1E2 $>90 \%$ ) and all gave similar phenotypes. Given that MO1 was most efficient in repressing expression of the gfi.1-GFP fusion gene, we used MO1 in most of our experiments.

In zebrafish, embryonic hematopoiesis begins at the 1 - to 2-somite stage (about $10 \mathrm{hpf}$ ) and blood circulation becomes visible at about $24 \mathrm{hpf}[19,20,23]$. Many studies have shown that hematopoiesis in zebrafish is controlled by conserved transcriptional regulators, which leads to progressive blood cell differentiation [19, 20, 23]. These factors include $\mathrm{Cdx} 4$, which is important for the initial determination of hematopoietic progenitors from the posterior lateral mesoderm (PLM) [24]; Scl and Lmo2, which are critical for the specification of all blood lineages [25-27]; C-myb, which is important for the development of both primitive and definitive erythroid cells [28]; Gata1, which promotes erythroid cell development and suppresses myeloid cell differentiation in zebrafish [29]; and Pu.1, which promotes myeloid cell development [30, 31]. Since GFI1 was identified as a transcriptional regulator that modulates many genes such as those that are critical for blood cell differentiation in mammals, we first analyzed the effects of gfil.1 downregulation on the expression of the transcription factors that are critical for blood cell development.

Whole-mount in situ hybridization results showed that the injection of MO1 reduced the expression of $s c l(83 \%$, $N=149)$ and $\operatorname{lmo} 2(81 \%, N=97)$. This reduction occurred primarily in the region around PLM that contains hematopoietic progenitor cells, and affected both the amount of expression and the size of the domain where the expression occurred (Figure 3A and 3B). In the ALM, the amount of $\mathrm{scl}$ and $\mathrm{lmo} 2$ expression was also reduced but the size of the expression domain was not affected (Figure $3 \mathrm{~A}$ and $3 \mathrm{~B})$. Furthermore, the expression of $c-m y b(83 \%, N=69)$ and gatal $(97 \%, N=191)$ was also reduced, showing much weaker staining of the bilateral hematopoietic cells that flank the paraxial mesoderm (Figure $3 \mathrm{C}$ and 3D). The effect of gfil.1 MO1 on gatal expression was also demonstrated by analyzing live gata 1:GFP transgenic zebrafish embryos ( $96 \%, N=213$, Figure $3 \mathrm{E})$. On the other hand, the expression of $p u .1$ was increased $(89 \%, N=78)$. The number of $p u .1$ positive cells in gfil.1 morphants is about 3 times more than that from control embryos as calculated using Image-Pro Plus 5.1 software (Figure 3F). However, gfil.1 MO1 had no effect on $c d x 4$ expression $(N=61$, Figure $3 \mathrm{G})$, which suggests a downstream role for $g$ fil.1 in relation to $c d x 4$.

The repressive effects of $g$ fil.1 on the expression of scl, lmo2, gatal, c-myb and pu.1 would probably lead to changes in the expression of their downstream genes and blood cell differentiation. We then investigated whether gfil.1 would affect lineage-specific marker gene expression at later developmental stages. These markers include l-plastin for macrophages of the embryonic myeloid lineage [32], mpo (myeloperoxidase) for granulocytes of the embryonic myeloid lineage [33], ikaros for lymphoid progenitors [34], hemoglobin alpha embryonic-1 ( hbae 1) for erythroid cells [35] and rag1 for T lymphocytes [34]. Consistent with the effect that $g$ fil.1 has on the expression of blood-specific transcription factors at about $12 \mathrm{hpf}$, injection of $g$ fil.1 MO1 downregulated the expression of hbael $(85 \%, N=121$, Figure 4A-4C), upregulated $l$-plastin $(92 \%, N=153$, Figure 4D), and did not affect ikaros ( $N=90$, Figure 4E). Interestingly, decreased expression of $m p o(90 \%, N=114$, Figure $4 \mathrm{~F})$ and $\mathrm{ragl}(80 \%, N=91$, Figure $4 \mathrm{G})$ was also detected in gfil.1 MO embryos. Since zebrafish contains multiple embryonic globin genes and hbael is just one of them, we further investigated the total amount of hemoglobin using $o$-dianisidine staining. As expected, the staining for hemoglobin was noticeably reduced in gfil.1 MO1-injected embryos at $84 \mathrm{hpf}(94 \%, N=157$, Figure $4 \mathrm{H})$. To elucidate the specificity of $g f i 1.1$ function, we tested the expression of etsrp, flk and flila as markers for blood vessels and pax2.1 as a marker for pronephric cells. In situ hybridization re- 

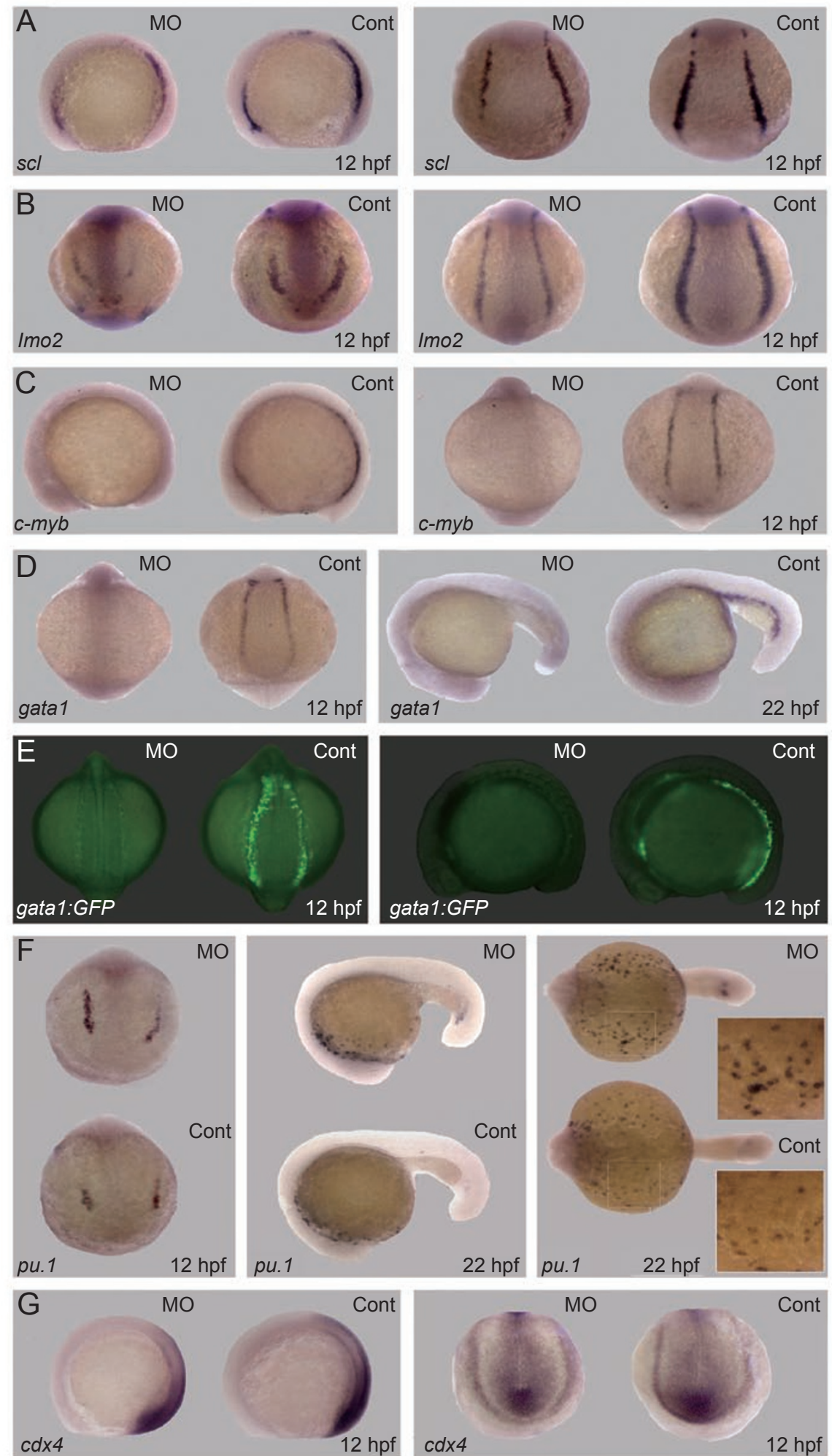

Figure 3 The effect of gfi1.1 knockdown on embryonic hematopoiesis. (A-F) Whole-mount in situ hybridization of scl (A), Imo2 (B), c-myb (C) and gata1 (D) at the indicated developmental stages of zebrafish embryos injected with $4 \mathrm{ng} \mathrm{MO} 1$ (MO) or the corresponding uninjected controls (Cont). (E) The effect of gfi1.1 MO1 (8 ng) on GFP expression in gata1:GFP transgenic fish. Whole-mount in situ hybridization of the pu.1 (F) and $c d x 4(\mathbf{G})$ genes at the indicated developmental stages of zebrafish embryos injected with $4 \mathrm{ng} \mathrm{MO1}(\mathrm{MO})$ or the corresponding uninjected controls (Cont). 

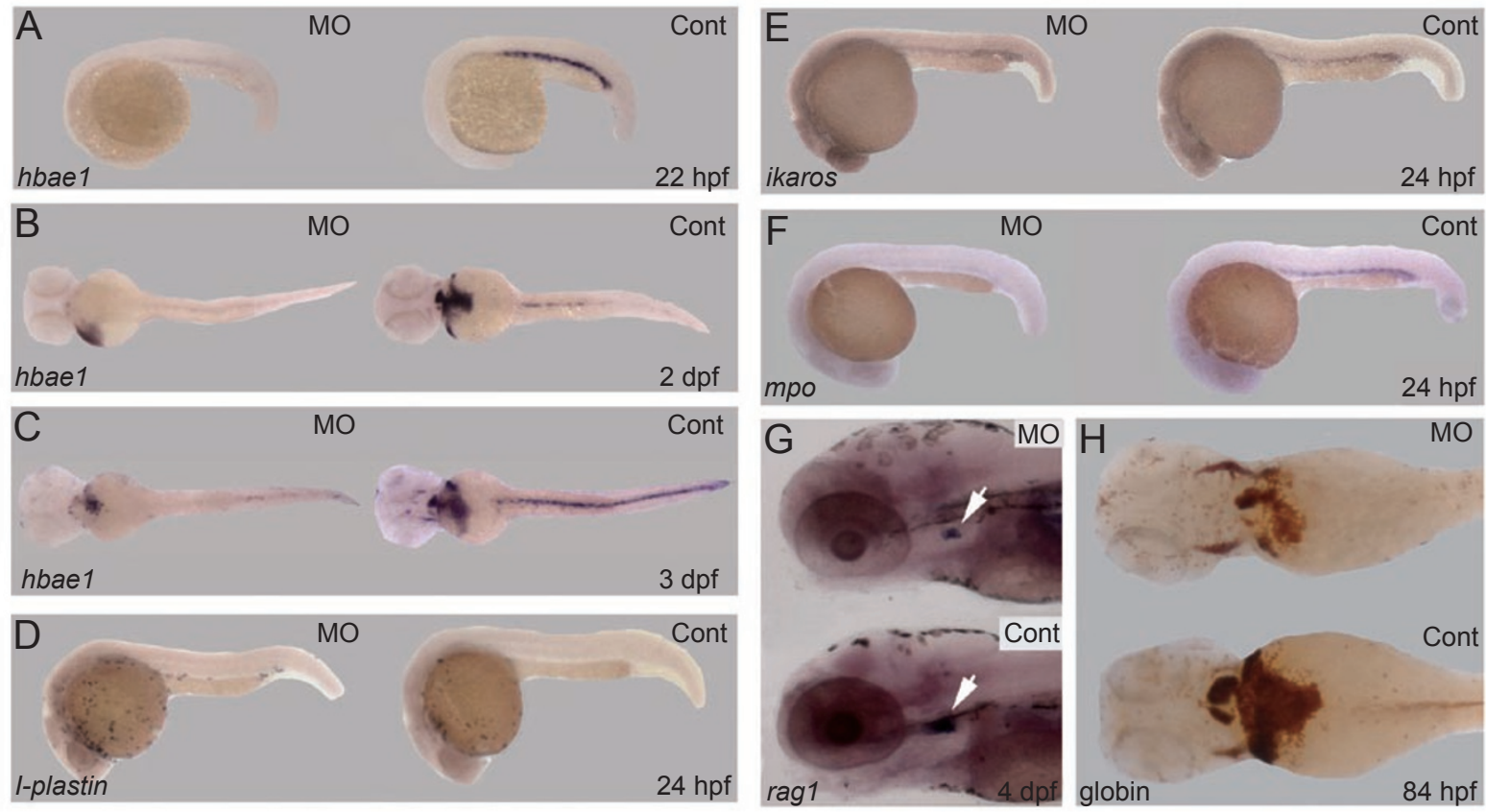

Figure 4 The effect of gfi1.1 knockdown on the differentiation of blood cells. Whole-mount in situ hybridization of $h b a e 1$ (A-C), I-plastin (D), ikaros (E), mpo (F) and rag1 (G) at the indicated developmental stages of zebrafish embryos injected with 4 ng MO1 (MO) or the corresponding uninjected controls (Cont). (H) Hemoglobin staining by o-dianisidine showing the erythrocytes that were affected by the application of gfi1.1 MO1 (4 ng).

sults showed no apparent differences in expression of these markers between gfil.1 MO1-injected and control embryos (Supplementary information Figure S3), which suggests that $g f i 1.1$ is specifically required for hematopoiesis because it promotes embryonic erythroid differentiation and represses myeloid differentiation. To further confirm our observation, we overexpressed gfil.1 by injecting $20 \mathrm{pg}$ of full-length mRNA per embryo; we detected increased gatal expression $(84 \%, N=90$, Figure $5 \mathrm{~A})$ but decreased pu. 1 expression $(90 \%, N=69$, Figure $5 \mathrm{~B})$.

\section{Discussion}

In this study, we have identified the zebrafish gfil.1 gene and have characterized its expression and biological function during embryonic hematopoiesis. Based on three lines of evidences, we believe that $g$ fil.1 represents the zebrafish ortholog of mammalian Gfil. First, zebrafish Gfil.1 protein has a conserved SNAG and six zinc-finger domains that are $95 \%$ and $98 \%$ similar to human GFI1, respectively. Second, there is a high similarity in syntenic arrangement of genes around the zebrafish $g$ fil.1 genomic locus compared with those around the human and mouse Gfil loci, where they share several homologous neighboring genes. Third, $g$ fil.1 is specifically expressed in hematopoietic tissues during embryonic development, which is consistent with the expression patterns seen in human and mouse Gfil.
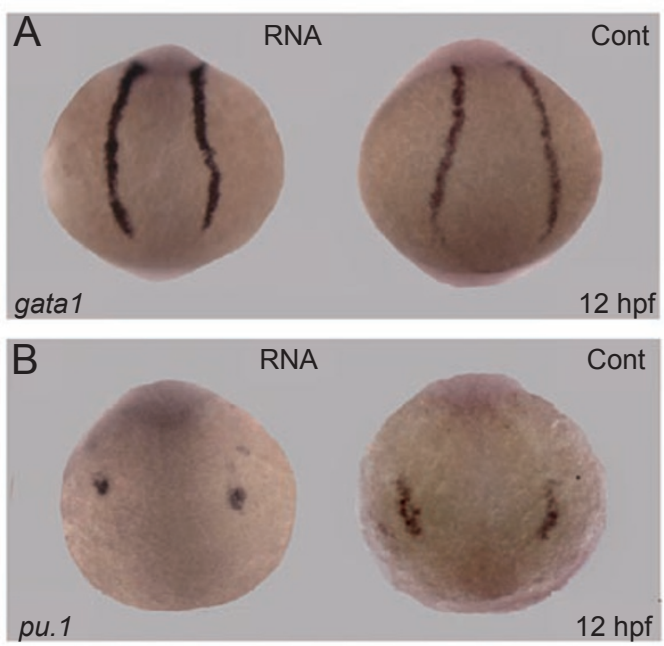

Figure 5 The effect of gfi1.1 overexpression on gata1 and pu.1 expression. Whole-mount in situ hybridization of gata1 (A) and pu. 1 (B) in the embryos injected with gfi1.1 mRNA (RNA) or the uninjected controls (Cont).

Our further studies have demonstrated that zebrafish Gfil.1 functions in promoting embryonic erythroid and repressing myeloid development. This finding is consistent with a recent report by Dahl et al. [36], which shows that mouse GFI1 antagonizes PU.1 activity during differentiation of hematopoietic progenitors. In zebrafish, we have 
shown in this study that the expression of transcription factors that are important for the erythroid lineage, including gatal, was reduced by knockdown of $g$ fil.1. However, we observed partial recovery of circulating red blood cells after 3 days of development, which suggests that the reduction did not lead to a complete block of erythroid cell development. This might be due to the functional redundancy of $g f$ genes in zebrafish. The mammalian GFI protein family includes three members: GFI1, GFI1B and GFI2. Both GFI1 and GFI1B contain an N-terminal SNAG domain and a C-terminal six zinc-finger domain and are mostly involved in hematopoiesis. Zebrafish seems to have two Gfil-like genes: $g$ fil.1, which is described in this study, and the previously reported $g f i 1$, which is mainly expressed in the ganglion cells of the neural retina and in developing hair cells of the ear. In addition, we have identified partial sequences of yet another gene that is related to the gfil family from the zebrafish genome and were able to detect its hematopoiesis-specific expression (unpublished data). It is possible that a more dramatic block of erythropoiesis may occur after a combined knockdown of all related hematopoiesis-specific gfil family genes in zebrafish.

Despite the fact that $g f i 1.1$ expression is detected throughout various stages from the zygote to $5 \mathrm{dpf}$, and that Gfil regulates many spatiotemporally expressed transcription factors that are critical for blood cell development, we propose that this gene is required in multiple hematopoietic processes. Zygotic expression of gfil.1 suggests that it represents one of the earliest hematopoiesis-specific genes and starts earlier than or at the same time as most of these transcription factors. In gfil.1 knockdown embryos, expression of the genes that are involved in the lineage differentiation of embryonic red blood cells, such as gatal, and of myeloid cells, such as pu.1, were affected. This indicates that during the early stages gfil.1 functions upstream of gatal and pu. 1 but downstream of $c d x 4$, as $c d x 4$ expression was not affected in $g$ fil.1 MO-injected embryos. Interestingly, decreased expression of mpo, which is a marker for granulocytes that have differentiated from the embryonic myeloid lineage, was detected in gfil.1 morphants. This result is consistent with the findings that Gfil-deficient mice appeared to be severely neutropenic and to have elevated immature monocytes in their blood and bone marrow [16], and suggests that $g$ fil.1 is also required for the differentiation of myeloid progenitor cells into granulocytes. Furthermore, gfil.1 knockdown also resulted in impaired expression of rag1, a marker gene for T lymphocytes, in 4 dpf embryos, although the expression of ikaros, a marker for lymphoid progenitor cells, was not affected in $24 \mathrm{hpf}$ embryos. We reasoned that $g f 1.1$ is most probably involved in lymphocyte differentiation by acting downstream of ikaros. The effects of gfil.1 on hematopoiesis seem to be specific as marker genes that are expressed in the closely related vascular and pronephric cells were not affected.

Mouse GFI1 carries out most of its functions through its transcriptional regulation activity and interacts with different targets in different cells [37]. In this study, gfil.1 knockdown affected the expression of several transcription factors that are critical for hematopoiesis, as well as some markers for differentiated blood cells. Gfil.1 may interact with these targets directly or indirectly. In gfil.1-deficient embryos decreased gatal but increased pu. 1 transcription was detected, which is consistent with the notion that $p u .1$ and gatal have antagonizing functions in the fate determination of hematopoietic cells between embryonic myeloid and erythroid cell lineages. This also suggests that Gfi1.1 is an upstream regulator of the process. Zebrafish gatal is likely one of the direct targets of gfil.1 since the gatal promoter region contains several putative conserved binding sites for Gfil family transcription regulators as predicated using ModelInspector software (data not shown). However, impaired gatal expression in gfil.1 MO-injected embryos may also be an indirect effect from the upregulation of pu.1. Thus, it would be interesting to identify more direct targets, isolate Gfil-interacting partners and determine how repression or activation is differentially achieved in different cells.

\section{Materials and Methods}

\section{Zebrafish gfil.1 gene identification and cDNA construction}

Transgenic zebrafish gfil.1:GFP was identified as a line that exhibits blood-specific expression of GFP from a large-scale enhancer trap screen (unpublished data) using a Tol2 vector [38] containing a gata2 minimal promoter linked to the GFP reporter gene [39]. Genomic sequences flanking this particular insertion were identified through linker-mediated PCR as described previously [40] and then mapped to specific zebrafish chromosomal loci using a blast search of the zebrafish Ensembl database (Jun 2007 Zv6). The gata 1: GFP transgenic fish line used in this study has been described previously [41]. Zebrafish were maintained at $28.5^{\circ} \mathrm{C}$ in a recirculating aquaculture system of the fish facility in Peking University.

For overexpression experiments, the full-length zebrafish $g$ fil.1 coding sequence was cloned into the $\mathrm{pCS} 2+$ vector containing the Sp6 promoter by reverse transcription-PCR using the following primers:

ATG Sense: 5'-ATG CCG AGG TCA TTT TTG-3'; Antisense: 5'-TTA TTT TAG CCC GTG CTG T-3'.

Gfi1.1 mRNA was produced through in vitro transcription following the manufacturer's instructions (Ambion).

The full-length coding sequence of $g f i 1.1$ was cloned into the $E G F P$-N1 vector (Clontech) to generate a $g$ fil.1-N1 plasmid encoding the Gfi1.1-EGFP fusion protein for detecting the efficiency of gfil.1 MO.

Reverse transcription and quantitative real-time PCR of gfil.1

Total RNA was extracted from 0-5 dpf embryos with TRIzol 
(Invitrogen) according to the manufacturer's instructions. Reverse transcription of purified RNA was performed using oligo (dT) priming and Superscript III reverse transcriptase (Invitrogen). The quantification of gene transcripts was done by qPCR, using the Brilliant SYBR Green QPCR Master Mix and Light Cycler apparatus (Stratagene). The primer pairs used were:

gfil.1: Sense: 5'-AGA AAG TCA AAC CGG ACA GG-3'; Antisense: 5'-AAA CGT ATG GAC GGA AGG AC-3'. actin: Sense: 5'-AGC AGA TGT GGA TCA GCAAG-3'; Antisense: 5'-AGT CAA TGC GCC ATA CAG AG-3'.

\section{Microinjection of $M O$}

Two ATG and one splicing MO against $g f i 1.1$ were ordered from Gene Tools, LLC. The MO sequences used were:

ATG MO1: 5'-GTA AAC ATG CCG AGG TCA TTT TTG G-3'; ATG MO2: 5'-GAG GCA ATA GTC GAA GTC CCA GTG G-3'; Splicing MO I1E2: 5'-GGT TAG ATC GGA CTT TTA CCG TGT T-3'. Control MO: 5'-CCT CTT ACC TCA GTT ACA ATT TAT A-3'.

The oligos were dissolved in water as a $25 \mathrm{mg} / \mathrm{ml}$ stock solution and injected at different dosages (4-10 ng) into 1- or 2-cell-stage embryos. Injected embryos were grown at $28.5{ }^{\circ} \mathrm{C}$ and observed under a microscope.

\section{$R N A$ whole-mount in situ hybridization}

RNA whole-mount in situ hybridization for gfil.1, scl, lmo2, gata1, pu.1, c-myb, hbae1, l-plastin, ikaros, mpo, ragl, etsrp, flk-1, fila and pax2.1 was performed as described previously [42]. The cDNA templates of these genes were amplified from total cDNA of 12 or 24 hpf embryos. Digoxigenin-UTP (Roche)-labeled antisense RNA probes were generated by in vitro transcription (Promega) and then purified (QIAGEN). All hybridization signals were detected via anti-digoxigenin-AP (Roche) and purple AP substrate (Promega).

\section{Hemoglobin staining}

Hemoglobin in zebrafish embryos was analyzed using $o$-dianisidine (Sigma) as described [35].

Gfil.1 antibody generation and detection by western blot analysis

An antibody against zebrafish Gfi1.1 was raised by immunizing C57/B6 mice with purified Gfil.1 protein expressed in Escherichia coli. Protein bands that were detected using the western blot technique were visualized by enhanced chemiluminescence. The infrared fluorescence image was obtained using the Odyssey infrared imaging system (Li-Cor Bioscience). The antibody against mouse Actin, which cross-reacts with zebrafish actin, was purchased from Sigma.

\section{Image acquisition and analysis}

For general examination, GFP-positive embryos or larvae were viewed under an Axioimager Z1 fluorescence microscope (Zeiss), equipped with $5 \times, 10 \times$ and $20 \times$ objectives, and a filter set for detection of GFP (excitation: 450-490 nm, barrier: $510 \mathrm{~nm}$, emission: 515-565 nm). Images of in situ hybridization results were taken under a Zeiss Stemi 2000-C microscope or an Axioimager A1 microscope (Zeiss).

\section{Acknowledgments}

We thank Koichi Kawakami for providing the Tol2 vector and Dr Yufeng Shi at Shanghai Institutes for Biological Science for critical discussions of this manuscript. We also thank Yan Zhuang for technical assistance and Yingdi Jia, Jingliang Chen and Houhua Cui for maintaining the zebrafish. This work was partially supported by the National Nature Science Foundation of China (No. 30721064, 30620120101 and J0630640), the 973 Program from the Ministry of Science and Technology of China (2005CB522504 and 2006CB943801) and the US National Institutes of Health (DK54508 to SL). BZ was supported by the New Century Excellent Talents in University and Excellent Young Teachers Program, Ministry of Education of China.

\section{References}

1 Hock H, Orkin SH. Zinc-finger transcription factor Gfi-1: versatile regulator of lymphocytes, neutrophils and hematopoietic stem cells. Curr Opin Hematol 2006; 13:1-6.

2 Zweidler-Mckay PA, Grimes HL, Flubacher MM, Tsichlis PN. Gfi-1 encodes a nuclear zinc finger protein that binds DNA and functions as a transcriptional repressor. Mol Cell Biol 1996; 16:4024-4034.

3 Grimes HL, Gilks CB, Chan TO, et al. The Gfi-1 protooncoprotein represses Bax expression and inhibits T-cell death. Proc Natl Acad Sci USA 1996; 93:14569-14573.

4 Gilks CB, Bear SE, Grimes HL, Tsichlis PN. Progression of interleukin-2 (IL-2)-dependent rat $\mathrm{T}$ cell lymphoma lines to IL-2-independent growth following activation of a gene (Gfi1) encoding a novel zinc finger protein. Mol Cell Biol 1993; 13:1759-1768.

5 Scheijen B, Jonkers J, Acton D, Berns A. Characterization of pal-1, a common proviral insertion site in murine leukemia virus-induced lymphomas of c-myc and Pim-1 transgenic mice. J Virol 1997; 71:9-16.

6 Zornig M, Schmidt T, Karsunky H, et al. Zinc finger protein GFI1 cooperates with myc and pim-1 in T-cell lymphomagenesis by reducing the requirements for IL-2. Oncogene 1996; 12:17891801.

7 Schmidt T, Karsunky H, Gau E, et al. Zinc finger protein GFI-1 has low oncogenic potential but cooperates strongly with pim and myc genes in T-cell lymphomagenesis. Oncogene 1998; 17:2661-2667.

8 Schmidt T, Karsunky H, Rodel B, et al. Evidence implicating Gfi-1 and Pim-1 in pre-T-cell differentiation steps associated with beta-selection. EMBO J 1998; 17:5349-5359.

9 Zhu J, Guo L, Min B, et al. Growth factor independent-1 induced by IL-4 regulates Th2 cell proliferation. Immunity 2002; 16:733744.

10 Rodel B, Tavassoli K, Karsunky H, et al. The zinc finger protein Gfi-1 can enhance STAT3 signaling by interacting with the STAT3 inhibitor PIAS3. EMBO J 2000; 19:5845-5855.

11 Person RE, Li FQ, Duan Z, et al. Mutations in proto-oncogene GFI1 cause human neutropenia and target ELA2. Nat Genet 2003; 34:308-312.

12 Saleque S, Cameron S, Orkin SH. The zinc-finger proto-oncogene Gfi-1b is essential for development of the erythroid and 
megakaryocytic lineages. Genes Dev 2002; 16:301-306.

13 Karsunky H, Zeng H, Schmidt T, et al. Inflammatory reactions and severe neutropenia in mice lacking the transcriptional repressor Gfil. Nat Genet 2002; 30:295-300.

14 Hock H, Hamblen MJ, Rooke HM, et al. Intrinsic requirement for zinc finger transcription factor Gfi-1 in neutrophil differentiation. Immunity 2003; 18:109-120.

15 Zeng H, Yucel R, Kosan C, et al. Transcription factor Gfil regulates self-renewal and engraftment of hematopoietic stem cells. EMBO J 2004; 23:4116-4125.

16 Hock H, Hamblen MJ, Rooke HM, et al. Gfi-1 restricts proliferation and preserves functional integrity of haematopoietic stem cells. Nature 2004; 431:1002-1007.

17 Phillips RL, Ernst RE, Brunk B, et al. The genetic program of hematopoietic stem cells. Science 2000; 288:1635-1640.

18 Weinstein BM, Schier AF, Abdelilah S, et al. Hematopoietic mutations in the zebrafish. Development 1996; 123:303-309.

19 de Jong JL, Zon LI. Use of the zebrafish system to study primitive and definitive hematopoiesis. Annu Rev Genet 2005; 39:481501.

20 Hsia N, Zon LI. Transcriptional regulation of hematopoietic stem cell development in zebrafish. Exp Hematol 2005; 33:10071014.

21 Dufourcq P, Rastegar S, Strahle U, Blader P. Parapineal specific expression of gfil in the zebrafish epithalamus. Gene Expr Patterns 2004; 4:53-57.

22 Nasevicius A, Ekker SC. Effective targeted gene 'knockdown' in zebrafish. Nat Genet 2000; 26:216-220.

23 Kimmel CB, Ballard WW, Kimmel SR, et al. Stages of embryonic development of the zebrafish. Dev Dyn 1995; 203:253-310.

24 Davidson AJ, Ernst P, Wang Y, et al. cdx4 mutants fail to specify blood progenitors and can be rescued by multiple hox genes. Nature 2003; 425:300-306.

25 Gering M, Rodaway AR, Gottgens B, et al. The SCL gene specifies haemangioblast development from early mesoderm. $E M B O$ $J$ 1998; 17:4029-4045.

26 Warren AJ, Colledge WH, Carlton MB, et al. The oncogenic cysteine-rich LIM domain protein rbtn2 is essential for erythroid development. Cell 1994; 78:45-57.

27 Green T. Haematopoiesis. Master regulator unmasked. Nature 1996; 383:575, 577.

28 Thompson MA, Ransom DG, Pratt SJ, et al. The cloche and spadetail genes differentially affect hematopoiesis and vasculogenesis. Dev Biol 1998; 197:248-269.

29 Galloway JL, Wingert RA, Thisse C, et al. Loss of gata1 but not gata2 converts erythropoiesis to myelopoiesis in zebrafish embryos. Dev Cell 2005; 8:109-116.

30 Hsu K, Traver D, Kutok JL, et al. The pu.1 promoter drives myeloid gene expression in zebrafish. Blood 2004; 104:12911297.

31 Lyons SE, Shue BC, Oates AC, et al. A novel myeloid-restricted zebrafish CCAAT/enhancer-binding protein with a potent transcriptional activation domain. Blood 2001; 97:2611-2617.

32 Crowhurst MO, Layton JE, Lieschke GJ. Developmental biology of zebrafish myeloid cells. Int J Dev Biol 2002; 46:483-492.

33 Bennett CM, Kanki JP, Rhodes J, et al. Myelopoiesis in the zebrafish, Danio rerio. Blood 2001; 98:643-651.

34 Langenau DM, Zon LI. The zebrafish: a new model of T-cell and thymic development. Nat Rev Immunol 2005; 5:307-317.

35 Detrich HW 3rd, Kieran MW, Chan FY, et al. Intraembryonic hematopoietic cell migration during vertebrate development. Proc Natl Acad Sci USA 1995; 92:10713-10717.

36 Dahl R, Iyer SR, Owens KS, et al. The transcriptional repressor GFI-1 antagonizes PU.1 activity through protein-protein interaction. J Biol Chem 2007; 282:6473-6483.

37 Kazanjian A, Gross EA, Grimes HL. The growth factor independence-1 transcription factor:new functions and new insights. Crit Rev Oncol Hematol 2006; 59:85-97.

38 Kawakami K, Takeda H, Kawakami N, et al. A transposon-mediated gene trap approach identifies developmentally regulated genes in zebrafish. Dev Cell 2004; 7:133-144.

39 Meng A, Tang H, Ong BA, et al. Promoter analysis in living zebrafish embryos identifies a cis-acting motif required for neuronal expression of GATA-2. Proc Natl Acad Sci USA 1997; 94:6267-6272.

$40 \mathrm{Wu}$ X, Li Y, Crise B, Burgess SM. Transcription start regions in the human genome are favored targets for MLV integration. Science 2003; 300:1749-1751.

41 Long Q, Meng A, Wang H, et al. GATA-1 expression pattern can be recapitulated in living transgenic zebrafish using GFP reporter gene. Development 1997; 124:4105-4111.

42 Jowett T, Lettice L. Whole-mount in situ hybridizations on zebrafish embryos using a mixture of digoxigenin- and fluorescein-labelled probes. Trends Genet 1994; 10:73-74.

(Supplementary Information is linked to the online version of the paper on the Cell Research website.) 